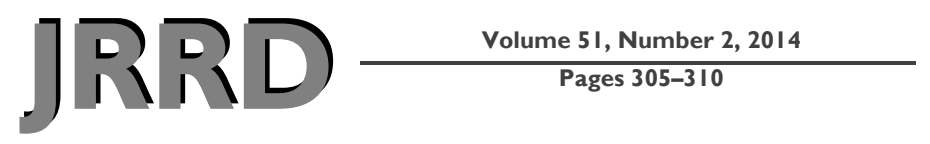

\title{
Detraining outcomes with expiratory muscle strength training in Parkinson disease
}

\author{
Michelle S. Troche, PhD; ${ }^{1-3^{*}}$ John C. Rosenbek, PhD; ${ }^{1-2}$ Michael S. Okun, MD; ${ }^{2,4}$ Christine M. Sapienza, PhD ${ }^{1-3}$ \\ ${ }^{1}$ Department of Speech, Language, and Hearing Sciences, and ${ }^{2}$ Center for Movement Disorders and Neurorestoration, \\ University of Florida, Gainesville, FL; ${ }^{3}$ Brain Rehabilitation Center, Malcom Randall Department of Veterans Affairs \\ Medical Center, Gainesville, FL; ${ }^{4}$ Departments of Neurology and Neurosurgery, University of Florida, Gainesville, $F L$
}

\begin{abstract}
Expiratory muscle strength training (EMST) is efficacious for improving maximum expiratory pressure (MEP), cough function, and swallowing safety in Parkinson disease (PD). However, there are no published reports describing detraining effects following EMST in persons with PD. Moreover, there are no published reports describing detraining effects following any behavioral swallowing intervention. Ten participants with PD underwent 3 mo of detraining following EMST. Measures of MEP and swallowing safety were made prior to beginning EMST (baseline), posttreatment (predetraining), and 3 mo postdetraining. Participants demonstrated, on average, a 19\% improvement in MEP from pre- to post-EMST. Following the 3 mo detraining period, MEP declined by $2 \%$ yet remained $17 \%$ above the baseline value. No statistically significant changes were found in swallowing safety from post-EMST to postdetraining period. Following the $3 \mathrm{mo}$ detraining period, seven participants demonstrated no change in swallowing safety, one worsened, and two had improvements. This preliminary study highlights the need for the design of maintenance programs to sustain function following intensive periods of training.
\end{abstract}

Key words: airway protection, aspiration, deglutition, detraining, dysphagia, expiratory muscle strength training, maximum expiratory pressure, Parkinson disease, swallowing, swallowing safety.

\section{INTRODUCTION}

Expiratory muscle strength training (EMST) with the EMST150 device (Aspire Products, LLC; Gainesville,
Florida) is a behavioral treatment paradigm used to increase expiratory and submental muscle force production [1-2]. EMST is efficacious for improving maximum expiratory pressure (MEP), cough effectiveness, and swallowing function [3-6]. The effects of 4 to $5 \mathrm{wk}$ of intensive EMST training are well described [3-4,6-11], but less is understood about the changes resulting from the discontinuation of its training. Detraining is the partial or complete loss of positive adaptations gained from the use of exercise training programs, which begins the moment an exercise paradigm is stopped [12-13]. The effects of EMST detraining are only documented for MEP and cough function in younger adults [7], older adults [6], and patients with multiple sclerosis (MS) [5,8] with reports of no reduction to 10 percent reduction in MEP over 4 to 8 wk of detraining. However, there are no

\footnotetext{
Abbreviations: $\mathrm{CME}=$ continuing medical education, $\mathrm{EMST}=$ expiratory muscle strength training, IRB = institutional review board, MEP = maximum expiratory pressure, $\mathrm{MS}=$ multiple sclerosis, NIH = National Institutes of Health, NPF $=$ National Parkinson Foundation, PAS = penetration-aspiration scale, $\mathrm{PD}=$ Parkinson disease, $\mathrm{PI}=$ primary investigator, $\mathrm{RCT}=$ randomized clinical trial, UF = University of Florida, VA = Department of Veterans Affairs, VAMC = Department of Veterans Affairs Medical Center.

*Address all correspondence to Michelle S. Troche, PhD; PO Box 117420, Gainesville, FL 32611; 352-273-3731; fax: 352-846-0243. Email: michi81@phhp.ufl.edu

http://dx.doi.org/10.1682/JRRD.2013.05.0101
} 
published reports describing EMST detraining in persons with Parkinson disease (PD). Moreover, there are no reports in the literature describing effects of detraining following any swallowing intervention. Information related to detraining following swallowing intervention is especially relevant in $\mathrm{PD}$, where patients often succumb to complications of dysphagia and resulting pulmonary sequelae [14-16]. The current study examined changes in MEP and swallowing safety following 3 mo of EMST detraining in persons with PD with the long-term objective of identifying targets for the development of robust impairment-specific maintenance programs.

\section{METHODS}

Recently, a blinded, placebo-controlled randomized clinical trial (RCT) involving 60 participants with PD was completed, testing the effects of 4 wk of EMST on swallowing and cough function (see Troche et al. [4]). Following completion of the experimental (active) arm of the aforementioned RCT ( $n=30$ [4]), 10 consecutive participants were offered enrollment in a detraining phase of study (University of Florida [UF] Institutional Review Board [IRB] 195-2005). The detraining phase lasted $3 \mathrm{mo}$. The sample size of 10 was selected as a convenience sample given the preliminary nature of the research question regarding detraining and the unknown effects of "no training" or "suspended training" on swallowing safety in PD. We selected the 3 mo detraining interval based on (1) previous studies assessing detraining in similar muscle groups [5-8], (2) minimization of participant attrition, and (3) reduction in time swallowing treatment was withheld.

MEP and swallowing safety data were collected using the same protocol completed in the RCT. Therefore, the same measures were completed prior to EMST (baseline), post-EMST (or predetraining), and following the 3 mo of detraining (postdetraining). All procedures were carried out with the adequate understanding and written consent of the participants involved and with the ethical approval of the UF and Malcom Randall Department of Veterans Affairs (VA) Medical Center (VAMC) IRBs. All participants were patients of the UF Center for Movement Disorders and Neurorestoration or the Malcom Randall VAMC Movement Disorders Clinic. A UF Movement Disorders fellowship trained neurologist completed a clinical assessment of each individual's PD disease severity (including blinded Unified PD Rating Scale ratings). Table 1 shows demographic information.

All participants with PD had to (1) meet the diagnostic criteria of the United Kingdom Brain Bank [17], (2) selfreport some degree of swallowing disturbance (i.e., reports of coughing with meals, increased eating duration), and (3) remain on the same PD medication regimen (including dosages and frequencies) throughout the study. Other inclusion criteria included (1) aged between 55 and 85 yr, (2) moderate clinical disability level (Hoehn \& Yahr stages II-IV), and (3) score of at least 24 on the Mini-Mental State Examination. Exclusion criteria, assessed by the recruiting neurologist prior to enrollment, included (1) other neurologic disorders, (2) gastrointestinal disease, (3) gastroesophageal surgery, (4) head and neck cancer, (5) history of breathing disorders or diseases, (6) untreated hypertension,

Table 1.

Participant demographics.

\begin{tabular}{|c|c|c|c|c|c|}
\hline \multirow{2}{*}{ Participant } & \multirow{2}{*}{ Age (yr) } & \multirow{2}{*}{ Sex } & \multirow{2}{*}{ Hoehn \& Yahr Stage } & \multicolumn{2}{|c|}{ Penetration-Aspiration Scale } \\
\hline & & & & Predetraining & Postdetraining \\
\hline 2 & 58 & M & 3 & 1 & 1 \\
\hline 4 & 74 & M & 3 & 5 & 2 \\
\hline 5 & 78 & M & 2.5 & 1 & 8 \\
\hline 8 & 71 & M & 3 & 2 & 2 \\
\hline 9 & 72 & $\mathrm{~F}$ & 3 & 1 & 1 \\
\hline 10 & 64 & $\mathrm{M}$ & 2 & 1 & 1 \\
\hline$\overline{M e a n} \pm$ SD & $70.0 \pm 6.4$ & - & - & - & - \\
\hline Median (Range) & - & - & $3(2-3.5)$ & $1.5(1-5)$ & $1.5(1-8)$ \\
\hline
\end{tabular}


(7) heart disease, (8) history of smoking in the last $5 \mathrm{yr}$, and (9) difficulty complying due to neuropsychological dysfunction (e.g., severe depression, psychosis).

\section{Testing Procedures and Outcomes}

\section{Primary Outcome: Maximum Expiratory Pressure}

Using a standardized protocol, participants were instructed to stand and occlude their nose with nose clips. MEP measurements were completed using a pressure manometer coupled to a mouthpiece via $50 \mathrm{~cm}$ and a $2 \mathrm{~mm}$ inner diameter tubing with an air leak created by a 14-gauge needle. The device was placed between the participants' lips and behind their teeth. They were then instructed to inhale as deeply as possible and blow into the manometer mouthpiece quickly and forcefully $[4,7,11,18]$. Solely verbal encouragement was provided to the participants. Three values within 5 percent of each other were required to achieve an average for the participants' individualized MEP score.

\section{Secondary Outcome: Swallowing Safety (Penetration- Aspiration Scale)}

Videofluoroscopy was used to examine swallowing function. Participants sat upright and their swallowing function was recorded in the lateral viewing plane using a properly collimated Phillips radiographic/fluoroscopic unit (63 kV, $1.2 \mathrm{~mA}$ output, full field of view mode). The Swallowing Signals Laboratory unit (Kay Elemetrics; Lincoln Park, New Jersey) digitally recorded the fluoroscopic images at 29.97 frames per second using a scan converter.

Participants completed ten 5 cc trials of thin liquid (Liquid E-Z Paque [barium sulfate] Suspension, E-Z-EM Inc; Lake Success, New York) (60\% w/v, 41\% w/w) by cup and also a trial of one $3 \mathrm{oz}$ sequential swallow of thin liquid by cup. Trials were randomly presented; therefore, the $3 \mathrm{oz}$ thin liquid sequential swallow could have taken place anywhere in the order of bolus presentations. During the swallowing examinations, all patients self-administered in order to approximate real-world feeding conditions. All participants were given the cup and prompted by the investigator to "place the liquid in your mouth and swallow when ready."

The selected swallowing outcome measure was the penetration-aspiration scale (PAS) [19], which was the same primary swallowing outcome measure from the RCT [4]. The PAS is a clinically relevant, validated, and ordinal measure, where 1 = safest swallow (no penetration or aspi- ration) and $8=$ least-safe swallow or silent aspiration. The scale measures whether or not material entered the airway, and if it entered the airway, whether the residue remained or was expelled. For the purposes of this study, the participants' worst PAS for the $3 \mathrm{oz}$ swallow was recorded and used as the measure of swallowing safety.

\section{Statistical Analysis}

Statistical analysis of the data was completed using SPSS (version 17.0, IBM Corporation; Armok, New York). Given the ordinal nature of the PAS and small sample size, a Wilcoxon signed rank test for two related samples was utilized to test differences in the two time points (post-EMST/predetraining and postdetraining) for the 10 participants in this detraining study.

\section{RESULTS}

\section{Primary Outcome: Maximum Expiratory Pressure}

Participants demonstrated, on average, a 19 percent improvement in MEP from pre- to post-EMST training (102.36-121.85 $\mathrm{cmH}_{2} \mathrm{O}, p=0.03$ ) (Figure). There was a 2 percent decrease in MEP (statistically nonsignificant) from post-EMST to postdetraining (121.85-119.4 $\mathrm{cmH}_{2} \mathrm{O}$ ), with average MEP scores postdetraining remaining 17 percent above the baseline (pre-EMST) values.

\section{Secondary Outcome: Swallowing Safety (Penetration- Aspiration Scale)}

No significant changes were found in PAS from postEMST to postdetraining period. To further probe the

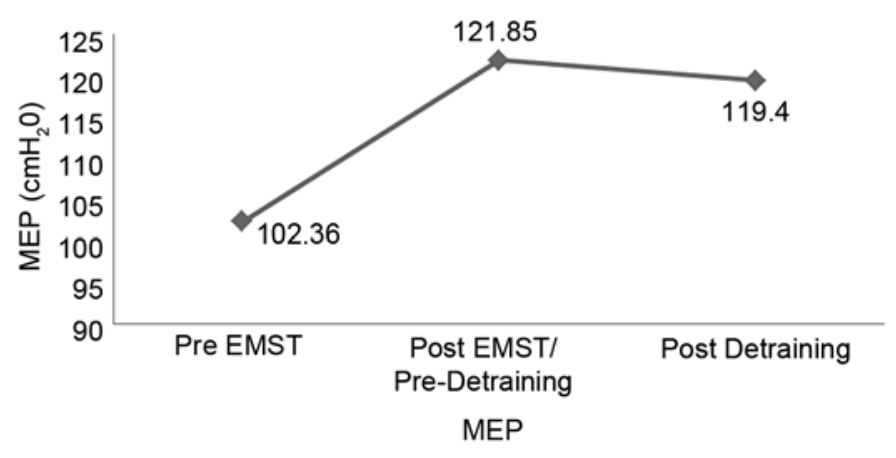

Figure.

Maximum expiratory pressure (MEP) at pre-expiratory muscle strength training (EMST), post-EMST or predetraining, and postdetraining time points for 10 participants included in detraining study. 
findings, the 10 participants were subdivided into those who had no change from post-EMST training to postdetraining, those who improved between time points, and those who worsened. Following the 3 mo detraining period, seven participants demonstrated no change in PAS, one had worsening of PAS, and two had improvements in PAS. Table 2 shows baseline (pre-EMST) and postdetraining median PAS scores by change group.

\section{DISCUSSION}

Strength training of the expiratory muscles is achieved, most typically, using a short-duration, musclespecific exercise with a handheld calibrated device [14,6-9]. EMST with the EMST150 device results in activation of submental and expiratory muscles $[11,18]$. The submental muscles play an important role in the superior and anterior movement of the hyolaryngeal complex during swallowing, therefore assisting in airway protection through the movement of the larynx under the base of the tongue and the opening of the upper esophageal sphincter [20-21]. In a large RCT (active EMST vs sham EMST), our research group tested the effects of EMST on swallowing function in participants with $\mathrm{PD}$, and results showed improvements in swallowing safety and cough airflow parameters after 4 wk of active EMST. More specifically, participants in the active EMST treatment group demonstrated increased MEP, reductions in PAS (i.e., safer swallowing), improved hyolaryngeal displacements, and improved cough volume acceleration [3-4]. There has been no other RCT assessing the efficacy of a restorative swallowing intervention in PD.

Therefore, the detraining-related outcomes reported here are critical to guiding this therapy into clinical practice, especially in light of the degenerative nature of PD. While the objective outcomes associated with the RCT were promising in persons with $\mathrm{PD}$, there remained questions about the physiological changes that occur when EMST is

Table 2.

Median penetration-aspiration scale (PAS) by change group.

\begin{tabular}{lcc}
\hline \multicolumn{1}{c}{ Change } & $\begin{array}{c}\text { Baseline PAS } \\
\text { (Pre-EMST) }\end{array}$ & $\begin{array}{c}\text { Postdetraining PAS } \\
\text { (Post-EMST Detraining) }\end{array}$ \\
\hline No Change & $5.0^{*}$ & 3.0 \\
Worsened & $8.0^{*}$ & 8.0 \\
Improved & $2.5^{*}$ & 1.5 \\
\hline ${ }^{*}$ Data from Troche et al. (2010) [4]. \\
EMST = expiratory muscle strength training. \\
\hline
\end{tabular}

discontinued. Previous detraining effects that have occurred in other populations have exclusively focused on breathing and cough function [5,7-8]. Baker et al. studied healthy participants who trained with EMST for 4 wk [7]. Following $8 \mathrm{wk}$ of detraining, MEP values remained significantly higher than baseline, with 7 to 10 percent loss. Chiara et al. studied the effects of $8 \mathrm{wk}$ of EMST in persons with MS and found that following 4 wk of detraining, no significant decrease in MEP was found and peak expiratory flow for cough remained significantly above baseline [5].

The current detraining study revealed a robust 19 percent improvement in MEP from pre- to post-EMST training in this group of participants. Participants only sustained a 2 percent decline in MEP following the detraining period The detraining effects on swallowing safety were less clear (Table 2), and this appears to be because baseline swallowing function (pre-EMST) across the individual participants was quite varied. For example, the participants who began EMST with the most impaired swallowing safety, although experiencing mild improvement in function with EMST, did not maintain the positive swallowing outcomes following the detraining period. Those with the least impaired swallowing safety pre-EMST training demonstrated a slight decrease in PAS (improvement) over the detraining period. This slight improvement is not considered clinically significant and likely demonstrates function within expected variability in swallowing performance. Participants with moderate deficits in swallowing safety prior to EMST maintained much of their benefit in swallowing safety following the 3 mo detraining period.

The detraining effects were not as predictable as hypothesized. It was expected that a detraining effect for MEP would occur when the EMST was suspended, and although there was a slight 2 percent decrease in MEP, this was not significant. It can be hypothesized that there was not a larger detraining effect to MEP due to the specificity of EMST on expiratory muscle force production, therefore solidifying the EMST training effect. Similarly, there was no significant detraining effect for swallowing safety. However, closer inspection of the swallowing data set revealed what appears to be an association with detraining that relates back to baseline (pre-EMST) swallowing functioning. Persons with more impaired swallowing detrained to a greater degree than persons with more functional swallowing at baseline. This supports the theory that reduced baseline motor coordination can result in greater detraining effect [13]. Future studies of EMST in PD will need to separate the baseline function for both MEP and swallowing 
impairment more carefully as well as include larger samples of persons with varying levels of dysfunction in order to more effectively answer this question regarding detraining.

\section{CONCLUSIONS}

Information related to detraining following dysphagia rehabilitation is highly relevant in populations with neurodegenerative disease where dysphagia and aspiration is often considered inevitable [22]. People with PD often succumb to complications of dysphagia and resulting pulmonary sequelae [14-16]. Therefore, long-term dysphagia management is a top priority throughout the progression of the disease. EMST has been found to provide specific, clinically meaningful gains in swallowing and cough [6]. This preliminary study highlights the need for the development of maintenance programs to sustain function following intensive periods of training.

\section{ACKNOWLEDGMENTS}

\section{Author Contributions:}

Research project conception: M. S. Troche, J. C. Rosenbek, M. S. Okun, C. M. Sapienza.

Research project organization and execution: M. S. Troche, C. M. Sapienza.

Drafting of manuscript: M. S. Troche. Critical review of manuscript for important intellectual content:

J. C. Rosenbek, M. S. Okun, C. M. Sapienza.

Statistical analysis: M. S. Troche, C. M. Sapienza.

Financial Disclosures: Dr. Okun serves as a consultant for the National Parkinson Foundation (NPF) and has received research grants from the National Institutes of Health (NIH), NPF, Michael J. Fox Foundation, Parkinson Alliance, Smallwood Foundation, and UF Foundation. Dr. Okun has previously received honoraria, but in more than 36 mo has received no support from industry, including travel. Dr. Okun has received royalties for publications with Demos, Manson, and Cambridge (movement disorders books). Dr. Okun has participated in continuing medical education (CME) activities on movement disorders sponsored by the University of South Florida CME office, PeerView, and Vanderbilt University. The institution and not Dr. Okun receives grants from Medtronic and the Academy of Natural Sciences/St. Jude, and the primary investigator (PI) has no financial interest in these grants. Dr. Okun has participated as a site PI and/or coinvestigator for several NIH-, foundation-, and industrysponsored trials over the years but has not received honoraria. Dr. Sapienza has previously received honoraria, including a consultant- ship with BAE Systems. Dr. Sapienza receives royalties from Plural Publishing. Dr. Sapienza has commercial interest in Aspire Products, LLC (which now manufactures the EMST150 device) but has received no direct funding from them for research. Aspire Products, LLC, had no involvement in study design; in the collection, analysis, and interpretation of data; in the writing of the article; or in the decision to submit the article for publication. Dr. Sapienza has participated as a coinvestigator for NIH, VA Rehabilitation Research and Development Service, and industry-sponsored trials over the years.

Funding/Support: This material was based on work supported by the VA Rehabilitation Research and Development Service (grant B6576-R) and the Michael J. Fox Foundation (grant 00056150). This work is also supported in part by an NIH (National Center for Advancing Translational Sciences) Clinical and Translational Science Award through the UF (UL1TR000064 and KL2TR000065) awarded to Dr. Troche.

Additional Contributions: The authors would like to thank the participants and their families. Additionally, they acknowledge the support of the NPF Center of Excellence at UF, the UF INFORM database, and the UF Foundation. Dr. Sapienza is now with the Department of Communication Sciences and Disorders, Jacksonville University, Jacksonville, Florida.

Institutional Review: All procedures were carried out with the adequate understanding and written consent of the participants involved and with the ethical approval of the UF and Malcom Randall VAMC IRBs (UF IRB 195-2005).

Participant Follow-Up: The authors do not plan to inform participants of the publication of this study.

\section{REFERENCES}

1. Wheeler KM, Chiara T, Sapienza CM. Surface electromyographic activity of the submental muscles during swallow and expiratory pressure threshold training tasks. Dysphagia. 2007;22(2):108-16. [PMID:17294298] http://dx.doi.org/10.1007/s00455-006-9061-4

2. Wheeler-Hegland KM, Rosenbek JC, Sapienza CM. Submental sEMG and hyoid movement during Mendelsohn maneuver, effortful swallow, and expiratory muscle strength training. J Speech Lang Hear Res. 2008;51(5): 1072-87. [PMID:18728114] http://dx.doi.org/10.1044/1092-4388(2008/07-0016)

3. Pitts T, Bolser D, Rosenbek J, Troche M, Okun MS, Sapienza CM. Impact of expiratory muscle strength training on voluntary cough and swallow function in Parkinson disease. Chest. 2009;135(5):1301-8. [PMID:19029430] http://dx.doi.org/10.1378/chest.08-1389

4. Troche MS, Okun MS, Rosenbek JC, Musson N, Fernandez HH, Rodriguez R, Romrell J, Pitts T, Wheeler-Hegland KM, Sapienza CM. Aspiration and swallowing in Parkinson 
disease and rehabilitation with EMST: A randomized trial. Neurology. 2010;75(21):1912-19. [PMID:21098406] http://dx.doi.org/10.1212/WNL.0b013e3181fef115

5. Chiara T, Martin AD, Davenport PW, Bolser DC. Expiratory muscle strength training in persons with multiple sclerosis having mild to moderate disability: Effect on maximal expiratory pressure, pulmonary function, and maximal voluntary cough. Arch Phys Med Rehabil. 2006;87(4):468-73. [PMID:16571384] http://dx.doi.org/10.1016/j.apmr.2005.12.035

6. Kim J, Davenport P, Sapienza C. Effect of expiratory muscle strength training on elderly cough function. Arch Gerontol Geriatr. 2009;48(3):361-66. [PMID:18457885] http://dx.doi.org/10.1016/j.archger.2008.03.006

7. Baker S, Davenport P, Sapienza C. Examination of strength training and detraining effects in expiratory muscles. J Speech Lang Hear Res. 2005;48(6):1325-33.

[PMID:16478374] http://dx.doi.org/10.1044/1092-4388(2005/092)

8. Chiara T, Martin D, Sapienza C. Expiratory muscle strength training: Speech production outcomes in patients with multiple sclerosis. Neurorehabil Neural Repair. 2007; 21(3):239-49. [PMID:17351085] http://dx.doi.org/10.1177/1545968306294737

9. Kim J, Sapienza CM. Implications of expiratory muscle strength training for rehabilitation of the elderly: Tutorial. J Rehabil Res Dev. 2005;42(2):211-24. [PMID:15944886] http://dx.doi.org/10.1682/JRRD.2004.07.0077

10. Sapienza C, Troche M, Pitts T, Davenport P. Respiratory strength training: Concept and intervention outcomes. Semin Speech Lang. 2011;32(1):21-30. [PMID:21491356] http://dx.doi.org/10.1055/s-0031-1271972

11. Silverman EP, Sapienza CM, Saleem A, Carmichael C, Davenport PW, Hoffman-Ruddy B, Okun MS. Tutorial on maximum inspiratory and expiratory mouth pressures in individuals with idiopathic Parkinson disease (IPD) and the preliminary results of an expiratory muscle strength training program. NeuroRehabilitation. 2006;21(1):71-79. [PMID:16720940]

12. Mujika I, Padilla S. Detraining: loss of training-induced physiological and performance adaptations. Part I: short term insufficient training stimulus. Sports Med. 2000; 30(2):79-87. [PMID:10966148]

http://dx.doi.org/10.2165/00007256-200030020-00002

13. Mujika I, Padilla S. Detraining: loss of training-induced physiological and performance adaptations. Part II: Long term insufficient training stimulus. Sports Med. 2000; 30(3):145-54. [PMID:10999420] http://dx.doi.org/10.2165/00007256-200030030-00001

14. Fernandez HH, Lapane KL. Predictors of mortality among nursing home residents with a diagnosis of Parkinson's disease. Med Sci Monit. 2002;8(4):CR241-46. [PMID:11951064]
15. Singer RB. Mortality in patients with Parkinson's disease treated with dopa. J Insur Med. 1992;24(2):126-27.

[PMID:10148480]

16. Gorell JM, Johnson CC, Rybicki BA. Parkinson's disease and its comorbid disorders: An analysis of Michigan mortality data, 1970 to 1990. Neurology. 1994;44(10):1865-68. [PMID:7936238] http://dx.doi.org/10.1212/WNL.44.10.1865

17. Hughes AJ, Daniel SE, Kilford L, Lees AJ. Accuracy of clinical diagnosis of idiopathic Parkinson's disease: A clinicopathological study of 100 cases. J Neurol Neurosurg Psychiatry. 1992;55(3):181-84. [PMID:1564476] http://dx.doi.org/10.1136/jnnp.55.3.181

18. Saleem AF, Sapienza CM, Okun MS. Respiratory muscle strength training: Treatment and response duration in a patient with early idiopathic Parkinson's disease. NeuroRehabilitation. 2005;20(4):323-33. [PMID:16403998]

19. Rosenbek JC, Robbins JA, Roecker EB, Coyle JL, Wood JL. A penetration-aspiration scale. Dysphagia. 1996;11(2): 93-98. [PMID:8721066] http://dx.doi.org/10.1007/BF00417897

20. Shaker R, Dodds WJ, Dantas RO, Hogan WJ, Arndorfer RC. Coordination of deglutitive glottic closure with oropharyngeal swallowing. Gastroenterology. 1990;98(6):1478-84. [PMID:2338189]

21. Matsuo K, Palmer JB. Anatomy and physiology of feeding and swallowing: Normal and abnormal. Phys Med Rehabil Clin N Am. 2008;19(4):691-707, vii. [PMID:18940636] http://dx.doi.org/10.1016/j.pmr.2008.06.001

22. Miller N, Allcock L, Hildreth AJ, Jones D, Noble E, Burn DJ. Swallowing problems in Parkinson disease: Frequency and clinical correlates. J Neurol Neurosurg Psychiatry. 2009;80(9):1047-49. [PMID:19028764] http://dx.doi.org/10.1136/jnnp.2008.157701

Submitted for publication May 1, 2013. Accepted in revised form September 19, 2013.

This article and any supplementary material should be cited as follows:

Troche MS, Rosenbeck JC, Okun MS, Sapienza CM. Detraining outcomes and expiratory muscle strength training in Parksinson disease. J Rehabil Res Dev. 2014; 51(2):305-10.

http://dx.doi.org/10.1682/JRRD.2013.05.0101

ResearcherID/ORCID: Michelle S. Troche, PhD: C-5205-2014

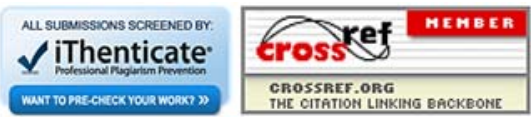

